\title{
Emergence of Mild Cognitive Impairment in Late Middle-Aged Adults in the Wisconsin Registry for Alzheimer's Prevention
}

\author{
Rebecca L. Koscik ${ }^{a}$ Asenath La Rue ${ }^{a}$ Erin M. Jonaitis ${ }^{a}$ \\ Ozioma C. Okonkwo ${ }^{b}$ Sterling C. Johnson ${ }^{a}$ b Barbara B. Bendlin ${ }^{b}$ \\ Bruce P. Hermann ${ }^{a, c}$ Mark A. Sager ${ }^{a, b}$ \\ a Department of Medicine, Wisconsin Alzheimer's Institute, ${ }^{b}$ Wisconsin Alzheimer's Disease \\ Research Center, and 'Department of Neurology, University of Wisconsin School of \\ Medicine and Public Health, Madison, Wis., USA
}

\section{Key Words}

Mild cognitive impairment · Longitudinal assessment $\cdot$ Cognition $\cdot$ Robust norms

\begin{abstract}
Aim: It is difficult to reliably detect the earliest signs of Alzheimer's disease (AD)-associated cognitive impairment. Our aim was to compare 3 psychometric methods of identifying amnestic mild cognitive impairment (aMCI) in a middle-aged longitudinal cohort enriched for AD risk. Methods: Wisconsin Registry for Alzheimer's Prevention (WRAP) participants with 3 waves of cognitive assessment over approximately 6 years were coded as meeting each of 3 psychometric aMCI definitions: (a) 'aMCI standard-baseline' used published norms to establish cutoffs for baseline performance; (b) 'aMCI robust-baseline' applied WRAP-specific robust norms to baseline, and (c) 'aMCI robust-multiwave' applied these robust norms across 3 waves of assessment. Each group was compared to a cognitively healthy subset. Results: Half the aMCI standard-baseline and one third of the aMCI robust-baseline group reverted to normal ranges at follow-up. Only the aMCI robust-multiwave method had an aMCI $\times$ age interaction showing significantly worse age-related memory declines in the aMCI group compared to the cognitively healthy group over 6 years of follow-up. Conclusion: Both cross-sectional methods showed instability over time, with many reverting to normal performance after baseline. The multiwave approach identified a group who showed progressive memory declines over 3 visits. Being able to detect progressive decline in late middle age is a critical step in improving prevention efforts.




\section{Introduction}

Dementia due to Alzheimer's disease (AD) is preceded by milder cognitive changes affecting episodic memory, executive function, or other cognitive skills [1,2]. The diagnostic category of mild cognitive impairment (MCI) was developed to define these changes and thus identify persons at risk for developing AD or other dementias [3]. Over the past decade, diagnostic criteria for MCI have undergone several revisions (e.g. Winblad et al. [4]), the most recent being the recommendations of a joint National Institute on Aging and Alzheimer's Association consensus panel [5]. The consensus panel focused on procedures to identify the symptomatic preclinical phase of $\mathrm{AD}$, referred to as $\mathrm{MCI}$ due to $\mathrm{AD}$. Clinical and cognitive criteria for $\mathrm{MCI}$ in general were specified as (a) concern about cognitive decline by the person or a significant other; (b) preservation of independence in functional abilities, and (c) impairment in 1 or more cognitive domains. For MCI due to AD, impairment in episodic memory was specified as the most likely early deficit, and the importance of obtaining evidence for progressive longitudinal cognitive decline was emphasized.

The leading model of AD pathogenesis based on the amyloid cascade [6] portrays cognitive change as a late-occurring event preceded by several years of clinically silent neurobiological changes. However, evidence from at-risk populations suggests that mild cognitive changes may be occurring in midlife and earlier in the cascade than previously believed. The Framingham offspring study [7] found reduced verbal and nonverbal memory performance among AD offspring at a mean age of 63 years; the differences in memory were significant among APOE $\varepsilon 4$ carriers only and were strongest in participants with a maternal family history of AD. Parental dementia was associated with worsening executive function over time, regardless of APOE genotype. In the Wisconsin Registry for Alzheimer's Prevention (WRAP), we found alterations in verbal list learning commonly associated with early-stage AD in middle-aged persons with a parental family history of AD, independent of APOE genotype $[8,9]$. Both studies have also shown atrophy in AD-sensitive brain regions in their middle-aged samples, most notably in persons with a parental family history of $\mathrm{AD}[7,10]$. Although neither the Framingham offspring study nor WRAP has linked these early cognitive and brain atrophy changes to increased risk of $A D$ as yet, previous investigations not selected for family history have shown associations between mild deficits in memory in middle age and increased risk of AD over intervals of 10 or more years $[11,12]$.

Because midlife may be a crucial phase of life for intervening to alter the trajectory of cognitive decline and the development of dementia, determining how best to identify middleaged persons at risk for developing $\mathrm{AD}$ is a high priority. One important step in this process is to determine whether current definitions of MCI effectively capture mild cognitive deficits occurring in middle age. While several studies have compared methods of operationalizing MCI criteria in older adults (e.g. Jak et al. [13]; Ganguli et al. [14]), only a handful of studies have applied MCI criteria to middle-aged samples, and these have shown mixed results regarding stability and correlates of MCI diagnoses. In 3 studies that included samples with mean ages in the $60 \mathrm{~s}$, the longitudinal stability of psychometrically identified MCI varied from $29 \%[15,16]$ to $70 \%$ [17], and none of the relatively young participants developed clinical dementia over follow-up intervals of 4-5 years. In the largest of these investigations (the Path Through Life Study) [15], a more broadly defined category of mild cognitive disorder, comprising any of several more specific diagnoses, showed a higher rate of stability across a 4-year interval than MCI per se. However, in the latest follow-up from this cohort [18], now young-old in age (68-72 years), more than $45 \%$ of mild cognitive disorder diagnoses were unstable across a period of 8 years. These initial investigations suggest that potentially significant cognitive impairments can be detected in midlife, but that single-point estimates of MCI may not be ideal for identifying the highest-risk individuals in this age span. This is important 
because biomarker profiles in preclinical AD may vary by the type of MCI definition employed in middle age.

The main aims of the present study were to compare the prevalences, baseline characteristics, and cognitive trajectories of 3 psychometric methods for identifying mild cognitive deficit in WRAP, a longitudinal study of over 1,500 late middle-aged persons at risk for developing dementia because of a parental family history of AD [19]. Given the centrality of declines in episodic memory in early-stage $\mathrm{AD}[1,5]$, we focused on amnestic $\mathrm{MCI}$ (aMCI), with or without other cognitive impairments. All 3 approaches entailed a psychometric classification of aMCI, supported by informant reports of minimal functional impairment and by medical history review. The first approach (aMCI standard-baseline) used published age-based norms [20] for a well-known episodic memory test to identify persons whose baseline scores were below expected levels. The second approach (aMCI robust-baseline) used robust norms specific to this sample to identify persons with episodic memory deficits at baseline in a manner conceptually similar to DeSanti et al. [21]. The third approach (aMCI robustmultiwave) implemented the recent recommendation of the joint consensus panel for longitudinal evidence of decline [5] and required episodic memory deficits (relative to robust norms) on multiple assessments spanning an average of 6 years.

Our analyses examined several hypotheses. First, we hypothesized that the aMCI standard-baseline approach would identify the fewest people with aMCI, while the robustbaseline approach would identify the most people. Second, to aid in interpreting whether memory deficits identified by each method reflect cognitive decline as opposed to stable lower ability, we hypothesized that each of the $3 \mathrm{aMCI}$ groups would be similar to the cognitively healthy $(\mathrm{CH})$ group in terms of estimates of premorbid cognitive abilities. Last, we hypothesized that the aMCI robust-multiwave approach would prove most effective in identifying persons with progressive memory change during the follow-up assessment window.

\section{Methods}

\section{Participants}

WRAP is a longitudinal study of a sample of over 1,500 middle-aged adults predominantly between the ages of 40 and 65 years at baseline. In order to increase our power to detect decline (and associated predictors) in middle age, the WRAP sample is enriched for a family history of AD, with over $70 \%$ of WRAP participants having a parent with either autopsy-confirmed or probable AD as defined by National Institute of Neurological and Communicative Disorders and Stroke and the Alzheimer's Disease and Related Disorders Association research criteria [22]. Follow-up assessments are underway, with second-wave assessments occurring approximately 4 years after baseline and all subsequent waves occurring at approximately 2-year intervals. As shown in figure 1, WRAP has a very low attrition rate, with less than $5 \%$ of the baseline sample being unavailable for cognitive follow-up (e.g. deceased or dropped out). A large subset of the baseline sample had not yet returned for their third-wave visit $(n=831,52.9 \%)$ and were therefore not included in analyses of our study hypotheses. To be included in testing the first hypothesis, participants must have completed 3 waves of testing, be free of dementia at or before the third wave of assessment, and be free of neurological conditions (including stroke, meningitis, epilepsy, multiple sclerosis, and Parkinson's disease); 532 met these inclusion/exclusion criteria (see sample flow chart in fig. 1). To be included in analyses of hypotheses 2 and 3, participants also had to meet aMCI criteria for 1 or more of the 3 psychometric aMCI approaches or meet criteria for ' $\mathrm{CH}$ ' across all 3 assessment waves.

\section{General Study Procedures}

Each wave of assessment includes a battery of commonly used clinical neuropsychological tests (see Sager et al. [19] for a description of the baseline cognitive battery), completion of questionnaires about health history and lifestyle, laboratory tests, and APOE genotyping. The neuropsychological battery included the measures used as cognitive outcomes for this study (see below), as well as the Mini-Mental State Examination (MMSE) [23], and estimated full-scale intelligence quotient (FSIQ) [24]. Questionnaires included measures 
Koscik et al.: Emergence of Mild Cognitive Impairment in Late Middle-Aged Adults in the Wisconsin Registry for Alzheimer's Prevention

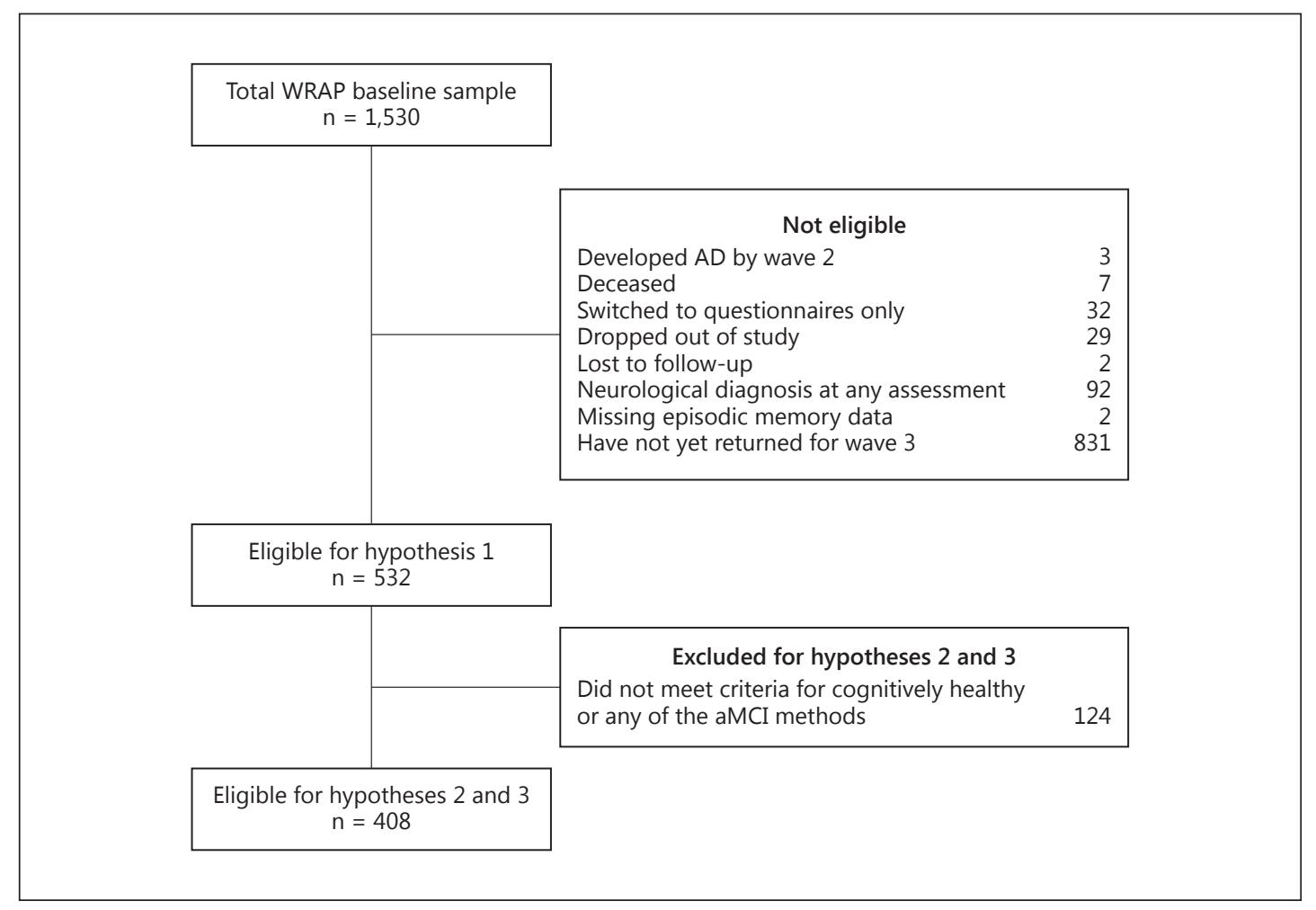

Fig. 1. Flow chart of WRAP participants who were eligible for this study.

of education, the Instrumental Activities of Daily Living scale (IADL) [25], and the Center for Epidemiologic Studies Depression scale (CES-D) [26]. All study procedures have been approved by the Health Sciences Institutional Review Board of the University of Wisconsin-Madison.

\section{Cognitive Variables}

Factor analysis using promax rotation and maximum likelihood estimation [27] was used to reduce the set of cognitive measures to a smaller number of factors and obtain weights used to combine the measures within each factor. The resulting 6 weighted factor scores were then standardized $(\sim N(0,1))$ into z-scores, using means and standard deviations (SDs) obtained from the whole baseline sample. Factors include 2 general ability indicators, Verbal Ability and Visuospatial Ability, comprised of the Wechsler Abbreviated Scale of Intelligence [24] subtests and related measures. There are 2 factors representing new learning and recall (Immediate Memory; Verbal Learning and Memory), both derived from the Rey Auditory Verbal Learning Test (AVLT) [28]. There are also 2 factors reflecting components of executive function (Working Memory, derived from the Digit Span Forward, Digit Span Backward, and Letter-Number Sequence subtests of the Wechsler Adult Intelligence Scale-III [29]; Speed and Flexibility, derived from Trails A, Trails B, and Stroop Color-Word). Additional details on the factor analysis methods and results can be found in Dowling et al. [30], Jonaitis et al. [31], and online supplementary Appendix A (for all online suppl. material, see www. karger.com/doi/10.1159/000355682) in this paper. The first 2 factors are obtained only at baseline and wave 2 , while the others, which are more likely to be sensitive to early cognitive decline, are obtained at all waves. Deficits in Immediate Memory or Verbal Learning and Memory are associated with aMCI and are the primary focus of this paper, while deficits in Working Memory or Speed and Flexibility are associated with nonamnestic MCI.

\section{Estimates of Premorbid Functioning}

Identifying performance that is indicative of subtle decline is challenging and requires appropriate population-based norms or individual baseline performance or both. Because baseline testing from earlier 
Koscik et al.: Emergence of Mild Cognitive Impairment in Late Middle-Aged Adults in the Wisconsin Registry for Alzheimer's Prevention

in life is generally lacking, demographically based prediction equations have been developed to estimate premorbid intellectual levels (e.g. Barona et al. [32]; Crawford et al. [33]; Griffin et al. [34]). Duff [35] and Duff et al. [36] have demonstrated that this approach can be extended to estimate premorbid memory abilities based on demographics and a measure of premorbid intellect; they further demonstrated that people with aMCI show significantly greater discrepancies between actual performance and estimated premorbid performance than $\mathrm{CH}$ peers. Adopting a similar approach, we used linear regression to develop prediction equations to estimate premorbid functioning for each of the aMCI-related factors using a combination of demographic variables [age, gender, and race (non-Hispanic Caucasian vs. other)] and premorbid intellect. Reading scores, used by Duff [35] and Duff et al. [36], have previously been shown to be reliable estimators of premorbid intellect $[37,38]$. Baseline Wide Range Achievement Test-III (WRAT-III) [39] raw reading scores were standardized using means and SDs from the WRAP baseline sample. Deciles from the standardized reading scores were then used in estimating premorbid functioning for the memory and executive function factors and in developing robust norms (as described in a subsequent section). Since the WRAP sample includes participants with siblings, the prediction equations for premorbid functioning used a subset of the baseline sample $(n=1,194)$ comprised of 1 randomly selected sibling per family to eliminate the influence of intra-family correlation on the regression coefficients; regression coefficients were then applied to the whole sample to obtain estimates of premorbid functioning corresponding to baseline age.

\section{Development of Robust Norms}

There are few published norms suitable for the relatively young age and high average education level of the WRAP cohort, especially on the AVLT, and those that are available lack information to assess sensitivity for identifying low performers in our sample. This problem has been addressed in other studies by developing robust internal norms (e.g. DeSanti et al. [21]; Sliwinski et al. [40]). Using the participants without a family history of $\mathrm{AD}$ as our reference group, we developed robust norms as follows. First, for each of the memory and executive function factors, we used baseline scores to develop lower prediction limits corresponding to a cutoff of -1.5 SDs below predicted based on age, gender, and WRAT reading decile; these are also sometimes referred to as 'conventional norms' (e.g. DeSanti et al. [21]). Those who had at least 1 factor score at or below the corresponding lower prediction limit at both baseline and wave $2(n=21)$ met exclusion criteria for preclinical decline and were removed, and the process was repeated on the reduced set to obtain new lower prediction limits; the new limits are referred to as 'robust norms'.

\section{Categorizing $\mathrm{CH}$ versus aMCI Subjects}

The factor scores from the memory domain were compared to the published and robust norms to categorize participants into either the $\mathrm{CH}$ group or an aMCI group for each of $3 \mathrm{aMCI}$ variables (aMCI standardbaseline, aMCI robust-baseline, and aMCI robust-multiwave). For all 3 aMCI variables, the CH group consists of participants who had no scores below published norms or robust norms at any of the first 3 assessment waves ( $\mathrm{n}=335$ ). The 'aMCI standard-baseline' method comes closest to the typical psychometric procedure for identifying possible aMCI, clinically, and historically, in research [3, 4] and includes people whose AVLT total or delayed scores at baseline fell at least 1.5 SDs below published norms $(n=24)$ [20]. Given that limitations in norms can lead to underrecognition of mild cognitive deficits, especially in persons with high education or premorbid ability [40,41], the 'aMCI robust-baseline' method includes people with 1 or more AVLT-based factor scores (Immediate Memory or Verbal Learning and Memory) falling at least 1.5 SDs below robust norms at baseline $(n=73)$. Finally, to reduce the odds of unstable classification frequently reported for nonclinical samples $[15,42,43]$, the 'aMCI robust-multiwave' group includes participants whose Immediate Memory and/or Verbal Learning and Memory factor scores fell below the robust norms on at least 2 of 3 waves of assessment $(n=61)$. For all categories, participants had normal-range performance on the MMSE (all had $\geq 25$ points, $90 \%$ had $\geq 28$ points) and informant reports of IADL function at wave 3 were also typically in the normal range ( $98 \%$ had $\geq 14$ points).

\section{Statistical Analyses}

Prevalence of aMCI in this sample was estimated for each aMCI method using a 95\% confidence interval (CI) and total sample size of $n=532$ (hypothesis 1). Sample characteristics were compared between groups (i.e. study sample vs. those yet to return for wave 3 and CH vs. each of the aMCI groups) using t tests for normally distributed data, two-sample Wilcoxon tests for continuous non-Gaussian data, and $\chi^{2}$ tests for categorical data. Estimates of premorbid functioning were also compared between the $\mathrm{CH}$ group and each of the aMCI groups using t tests (hypothesis 2). Longitudinal performance on each of the memory and executive 
Koscik et al.: Emergence of Mild Cognitive Impairment in Late Middle-Aged Adults in the Wisconsin Registry for Alzheimer's Prevention

Fig. 2. Overlap between aMCI methods: circles are approximately proportional to overall percentage of those meeting aMCI criteria for 1 or more methods.

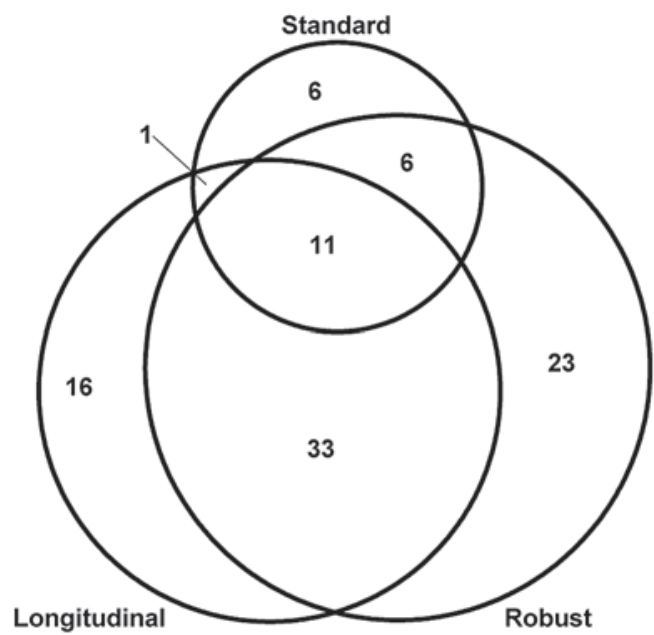

function factors was compared across $\mathrm{CH}$ and aMCI groups using linear mixed models adjusting for baseline age, gender, and WRAT reading; possible random effects included family, individual, and individual slope. This approach to modeling facilitates comparisons of between-group changes, while also adjusting for intraindividual correlations [44]. For each factor and aMCI method, the model including the aMCI method $\times$ age interaction was examined first to test whether rates of cognitive decline differed between the $\mathrm{CH}$ and aMCI groups (hypothesis 3). If the aMCI $\times$ age interaction was significant, indicating differing rates of decline between $\mathrm{CH}$ and $\mathrm{aMCI}$, separate simple age intercepts and slopes were calculated for the $\mathrm{CH}$ and aMCI groups using the intercept, aMCI, and age parameters from the regression model [45]. If the aMCI $\times$ age interaction was not significant $(\mathrm{p} \geq 0.05)$, the interaction term was removed and the aMCI main effect was examined.

In secondary analyses, since APOE $\varepsilon 4$ carrier status and a positive family history of AD increase risk of $\mathrm{AD}$, we also examined the interaction of each of these with age in parallel models (i.e. same covariates and random effects). We also used $\chi^{2}$ tests to test whether each of these was independent of each of the 3 aMCI variables. All analyses were performed using SAS v.9.3; all statistical tests used an $\alpha$ of 0.05 .

\section{Results}

A total of 532 participants (98.7\% non-Hispanic Caucasians) met inclusion criteria for the first hypothesis examining prevalence of aMCI across the 3 psychometric classification methods. The study sample was similar in gender and APOE $\varepsilon 4$ carrier status to those who were eligible except for not having returned yet for their third visit $(n=831$ in fig. 1). The study sample was more non-Hispanic Caucasian, younger, had more people with a college degree and family history of $\mathrm{AD}$, had fewer people with elevated depression scores, and performed better on baseline assessment of IQ and literacy than those who have yet to return for a third visit. Details of the comparisons of these baseline characteristics are presented in online supplementary Appendix B.

\section{Hypothesis 1}

In the study sample ( $\mathrm{n}=532)$, the percentage meeting aMCI criteria (and corresponding $95 \% \mathrm{CI}$ ) was $4.5 \%$ (2.7-6.3) for the aMCI standard-baseline approach, 13.7\% (10.8-16.6) for the aMCI robust-baseline method, and $11.5 \%$ (8.8-14.2) for the aMCI robust-multiwave method. A total of $96(18 \%)$ people in the sample met aMCI criteria for 1 or more methods; overlap in the 3 methods is shown in figure 2 . 
Table 1. Comparison of sample characteristics and premorbid functioning: $\mathrm{CH}$ versus each aMCI group

\begin{tabular}{|c|c|c|c|c|}
\hline Sample characteristics & $\begin{array}{l}\mathrm{CH} \\
(\mathrm{n}=335)\end{array}$ & $\begin{array}{l}\text { aMCI standard- } \\
\text { baseline } \\
(n=24)\end{array}$ & $\begin{array}{l}\text { aMCI robust- } \\
\text { baseline } \\
(n=73)\end{array}$ & $\begin{array}{l}\text { aMCI robust- } \\
\text { multiwave } \\
(\mathrm{n}=61)\end{array}$ \\
\hline Age at baseline, years & $52.5 \pm 6.6$ & $52.8 \pm 5.4$ & $53.6 \pm 6.6$ & $54.7 \pm 5.6^{*}$ \\
\hline Age at visit 3 , years & $59.0 \pm 6.4$ & $59.3 \pm 5.4$ & $60.2 \pm 6.4$ & $61.3 \pm 5.5^{*}$ \\
\hline Female gender & $240(71.6)$ & $11(45.8)^{\dagger}$ & $48(65.8)$ & $38(62.3)$ \\
\hline \multicolumn{5}{|l|}{ Education } \\
\hline$<$ College & $28(8.4)$ & $1(4.2)$ & $6(8.2)$ & $2(3.3)$ \\
\hline Some college & $99(29.6)$ & $10(41.7)$ & $23(31.5)$ & $19(31.2)$ \\
\hline$\geq$ College degree & $208(62.1)$ & $13(54.2)$ & $44(60.3)$ & $40(65.6)$ \\
\hline APOE $\varepsilon 4$ carrier & $143(42.7)$ & $8(33.3)$ & $23(31.5)$ & $19(31.2)$ \\
\hline Family history of AD & $273(81.5)$ & $21(87.5)$ & $59(80.8)$ & $47(77.1)$ \\
\hline FSIQ & $114.7 \pm 9.2$ & $109.4 \pm 8.6^{\dagger}$ & $111.9 \pm 9.9^{*}$ & $113.3 \pm 9.7$ \\
\hline WRAT reading decile & $0.10 \pm 0.9$ & $-0.32 \pm 0.8^{*}$ & $0.25 \pm 0.9$ & $0.44 \pm 0.9^{\dagger}$ \\
\hline Baseline CES-D $\geq 16$ & $23(6.9)$ & $1(4.2)$ & $7(9.6)$ & $8(13.1)$ \\
\hline \multicolumn{5}{|l|}{ Estimates of premorbid functioning } \\
\hline Immediate Memory & $0.10 \pm 0.3$ & $-0.12 \pm 0.3^{\dagger}$ & $0.07 \pm 0.3$ & $0.09 \pm 0.3$ \\
\hline Verbal Learning and Memory & $0.04 \pm 0.4$ & $-0.26 \pm 0.1^{\dagger}$ & $0.01 \pm 0.5$ & $0.04 \pm 0.4$ \\
\hline Speed and Flexibility & $0.14 \pm 0.4$ & $-0.03 \pm 0.4^{*}$ & $0.06 \pm 0.4$ & $0.06 \pm 0.3$ \\
\hline Working Memory & $0.03 \pm 0.4$ & $-0.15 \pm 0.4^{*}$ & $0.08 \pm 0.4$ & $0.17 \pm 0.4^{*}$ \\
\hline
\end{tabular}

Values are means \pm SD or numbers with percentages in parentheses. ${ }^{*} p<0.05 ;{ }^{\dagger} p<0.01 ; p$ values from $\mathrm{t}$ test for variables with mean $\pm \mathrm{SD}$ and $\chi^{2}$ for categorical variables.

Stability of aMCI Baseline Classifications. Of the 24 participants who met aMCI standardbaseline criteria, more than one half $(\mathrm{n}=14,58.3 \%)$ reverted to the normal range at subsequent visits, slightly less than one fifth $(n=4,16.7 \%)$ fell at least 1.5 SDs below published norms (i.e. met aMCI standard criteria) at all 3 visits, and the remainder showed inconsistent patterns across the 3 testings [ 1 participant (4.2\%) met aMCI standard criteria at wave 2 but not wave 3 , and 5 participants $(20.8 \%)$ reverted to normal at wave 2 but met aMCI standard criteria again at wave 3]. Of the 73 participants who met aMCI robust-baseline criteria, 29 $(39.7 \%)$ reverted to the normal range at subsequent visits, nearly one third met criteria at all 3 visits $(\mathrm{n}=24,32.9 \%)$, and the remainder varied across testings [8 participants $(11.0 \%)$ met aMCI robust criteria at waves 1 and 2 only, and 12 participants (16.4\%) met aMCI robust criteria at waves 1 and 3 only].

\section{Hypotheses 2-3}

The remainder of our analyses compared the 335 participants who met psychometric criteria for $\mathrm{CH}$ with the aMCI group from each of the 3 classification methods. Subjects whose performance did not meet criteria for $\mathrm{CH}$ or 1 or more aMCI variables were omitted from these analyses. Table 1 summarizes sample characteristics for each group. The aMCI standardbaseline group differs significantly from the $\mathrm{CH}$ group in the following ways: it has more men, lower baseline IQ and WRAT reading scores, and lower estimates of premorbid functioning for all 4 factors representing memory and executive function. The aMCI robust-baseline group differs significantly from the $\mathrm{CH}$ group only in terms of baseline IQ. The aMCI robustmultiwave group is older, has higher baseline reading scores, and higher baseline Working Memory scores than the $\mathrm{CH}$ group. 
Koscik et al.: Emergence of Mild Cognitive Impairment in Late Middle-Aged Adults in the Wisconsin Registry for Alzheimer's Prevention

Table 2. Parameter estimates from linear mixed models by aMCI method

\begin{tabular}{|c|c|c|c|c|}
\hline & \multicolumn{2}{|l|}{ Memory factors } & \multicolumn{2}{|c|}{ Executive function factors } \\
\hline & $\begin{array}{l}\text { Immediate } \\
\text { Memory } \\
\beta(\mathrm{SE})\end{array}$ & $\begin{array}{l}\text { Verbal Learning } \\
\text { and Memory } \\
\beta(\mathrm{SE})\end{array}$ & $\begin{array}{l}\text { Speed and } \\
\text { Flexibility } \\
\beta(\mathrm{SE})\end{array}$ & $\begin{array}{l}\text { Working } \\
\text { Memory } \\
\beta(\text { SE) }\end{array}$ \\
\hline \multicolumn{5}{|l|}{ aMCI standard-baseline } \\
\hline Intercept & $-0.55(0.130)^{\ddagger}$ & $-1.30(0.130)^{\ddagger}$ & $0.25(0.170)$ & $0.14(0.160)$ \\
\hline Age, years ${ }^{\mathrm{a}}$ & $-0.019(0.004)^{\ddagger}$ & $0.027(0.016)^{\ddagger}$ & $-0.016(0.004)^{\dagger}$ & $-0.007(0.004)$ \\
\hline Reading decile & $0.34(0.035)^{\ddagger}$ & $0.29(0.037)^{\ddagger}$ & $0.19(0.047)^{\ddagger}$ & $0.54(0.044)^{\ddagger}$ \\
\hline Male gender & $-0.56(0.067)^{\ddagger}$ & $-0.48(0.070)^{\ddagger}$ & $-0.33(0.090)^{\dagger}$ & $0.010(0.084)$ \\
\hline aMCI standard $($ status $=\mathrm{CH})$ & $1.12(0.12)^{\ddagger}$ & $1.91(0.13)^{\ddagger}$ & $0.33(0.17)^{*}$ & $0.12(0.16)$ \\
\hline aMCI standard $\times$ age & $-0.015(0.018)$ & $-0.041(0.016)^{*}$ & $0.020(0.019)$ & $-0.00003(0.016)$ \\
\hline \multicolumn{5}{|l|}{ aMCI robust-baseline } \\
\hline Intercept & $-0.75(0.072)^{\ddagger}$ & $-0.82(0.080)^{\ddagger}$ & $-0.055(0.098)$ & $-0.34(0.090)^{\dagger}$ \\
\hline Age, years ${ }^{\mathrm{a}}$ & $-0.020(0.004)^{\ddagger}$ & $-0.012(0.004)^{\dagger}$ & $-0.016(0.004)^{\dagger}$ & $-0.006(0.004)$ \\
\hline Reading decile & $0.31(0.032)^{\ddagger}$ & $0.29(0.036)^{\ddagger}$ & $0.15(0.044)^{\dagger}$ & $0.51(0.041)^{\ddagger}$ \\
\hline Male gender & $-0.55(0.063)^{\ddagger}$ & $-0.53(0.070)^{\ddagger}$ & $-0.35(0.085)^{\ddagger}$ & $0.027(0.079)$ \\
\hline aMCI robust $($ status $=\mathrm{CH}$ ) & $1.32(0.074)^{\ddagger}$ & $1.43(0.083)^{\ddagger}$ & $0.63(0.100)^{\ddagger}$ & $0.59(0.094)^{\ddagger}$ \\
\hline aMCI robust $\times$ age & $-0.001(0.010)$ & $-0.012(0.010)$ & $0.006(0.011)$ & $-0.008(0.010)$ \\
\hline \multicolumn{5}{|l|}{ aMCI robust-multiwave } \\
\hline Intercept & $-0.84(0.095)^{\ddagger}$ & $-1.01(0.090)^{\ddagger}$ & $-0.26(0.110)^{*}$ & $-0.41(0.101)^{\ddagger}$ \\
\hline Age, years ${ }^{\mathrm{a}}$ & $-0.044(0.011)^{\ddagger}$ & $-0.037(0.010)^{\dagger}$ & $-0.016(0.004)^{\dagger}$ & $-0.004(0.004)$ \\
\hline Reading decile & $0.33(0.033)^{\ddagger}$ & $0.32(0.034)^{\ddagger}$ & $0.18(0.045)^{\ddagger}$ & $0.53(0.042)^{\ddagger}$ \\
\hline Male gender & $-0.53(0.063)^{\ddagger}$ & $-0.49(0.066)^{\ddagger}$ & $-0.31(0.086)^{\dagger}$ & $-0.010(0.080)$ \\
\hline aMCI longitudinal $($ status $=\mathrm{CH})$ & $1.40(0.098)^{\ddagger}$ & $1.62(0.092)^{\ddagger}$ & $0.83(0.110)^{\ddagger}$ & $0.68(0.100)^{\ddagger}$ \\
\hline aMCI longitudinal $\times$ age & $0.024(0.011)^{*}$ & $0.022(0.010)^{*}$ & $0.001(0.012)$ & $-0.020(0.010)$ \\
\hline
\end{tabular}

${ }^{a}$ Age is centered around a baseline median of 54 years. If the interaction was not significant, the model was rerun without the interaction term, and parameter estimates reflect the model with the aMCI main effect only.

${ }^{*} \mathrm{p}<0.05 ;{ }^{\dagger} \mathrm{p}<0.01 ;^{\ddagger} \mathrm{p}<0.0001$.

\section{Cognitive Decline in $\mathrm{CH}$ versus aMCI Groups}

For each aMCI method and cognitive outcome, the first model examined included random effects, covariates, aMCI method, and the aMCI method $\times$ age interaction. When the interaction was not significant, it was removed and the model was rerun. Parameter estimates of the resulting 12 mixed effects models are summarized in table 2. For the aMCI standardbaseline analyses, the only significant aMCI $\times$ age interaction was for the Verbal Learning and Memory factor. Simple age slopes, calculated as described in table 2 and shown in figure 3a, indicate an interaction opposite to what one would expect of criteria that reliably identify decliners. Specifically, the analysis showed an age-related slope of -0.014 SDs/year in the CH group compared with an age-related slope of $0.027 \mathrm{SDs} /$ year in the aMCI standard group. Thus, published norms identified a group of people whose verbal learning started lower but improved, while those in the $\mathrm{CH}$ group started higher and showed modest age-related decline. aMCI standard-baseline main effects were significant for Immediate Memory and Speed and Flexibility, with the $\mathrm{CH}$ group showing better performance than the aMCI group in both factors.

For the aMCI robust-baseline analyses, there were no significant aMCI $\times$ age interactions, and all aMCI main effects were significant, with the $\mathrm{CH}$ group scoring higher than the aMCI 
Dementia

and Geriatric
Dement Geriatr Cogn Disord 2014;38:16-30

DOI: $10.1159 / 000355682$

Www.karger.com/dem

Koscik et al.: Emergence of Mild Cognitive Impairment in Late Middle-Aged Adults in the Wisconsin Registry for Alzheimer's Prevention
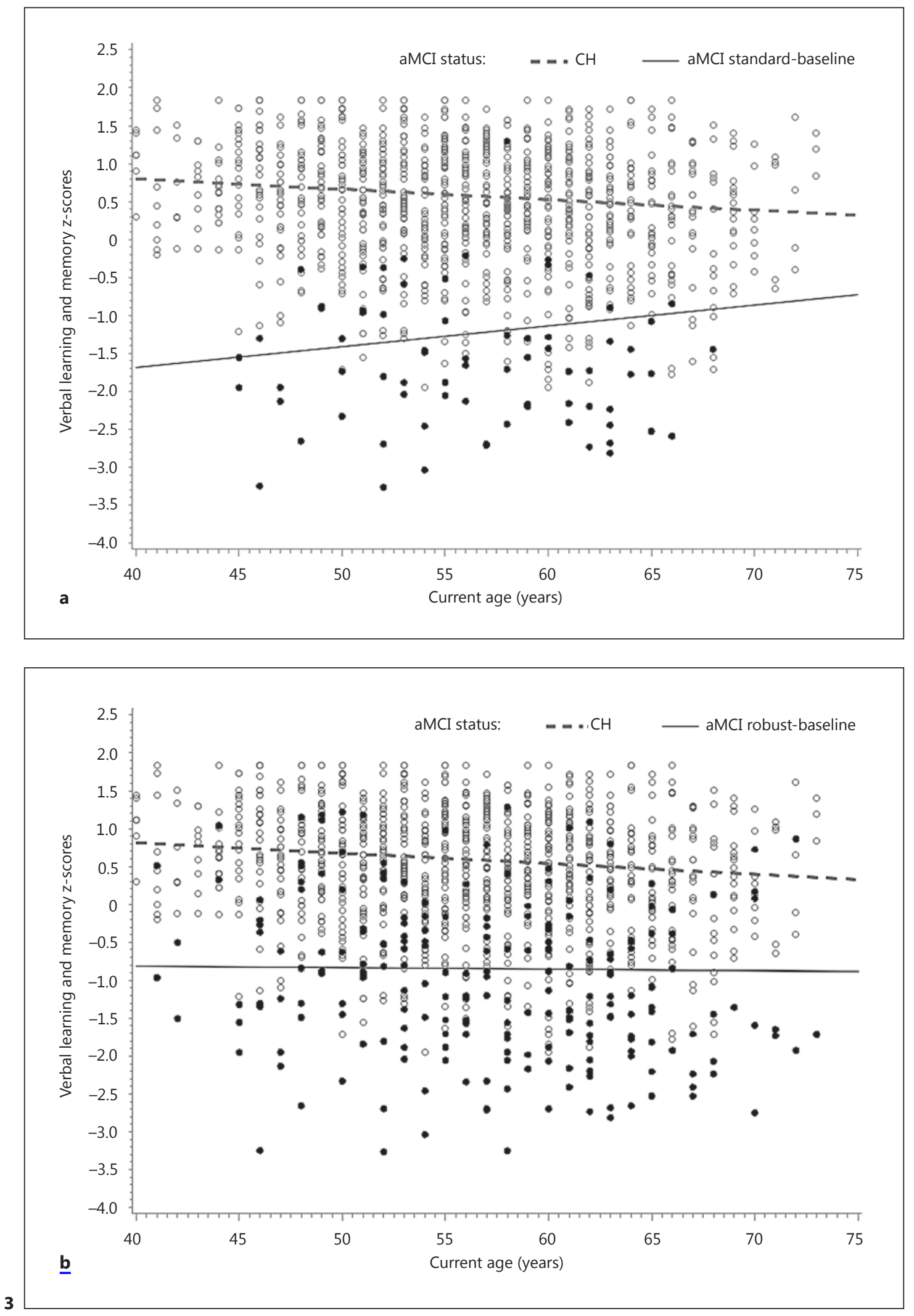

(For Legend see next page.) 


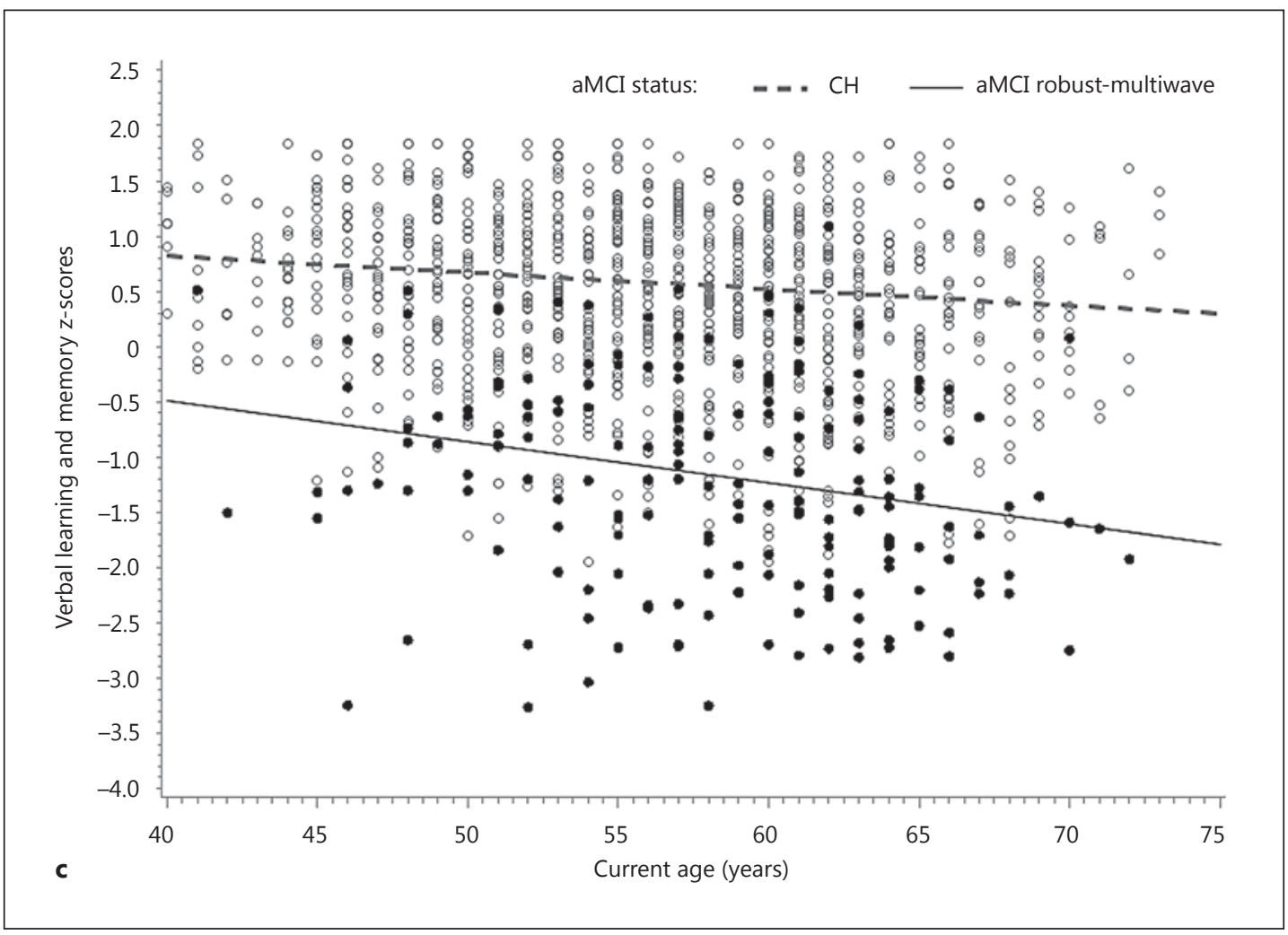

Fig. 3. Verbal learning and memory factor scores versus age at assessment for the $\mathrm{CH}$ group versus 1 of the aMCI methods. Each person has 3 data points represented in the figure. The simple slopes are calculated using results of the mixed effects models presented in table 2: aMCI intercept = model intercept; aMCI age slope $=$ age $\beta ; \mathrm{CH}$ intercept $=$ model intercept $+\mathrm{aMCI}(=\mathrm{CH}) \beta ; \mathrm{CH}$ age slope $=$ age slope + interaction $\beta$ (i.e. these are calculated for the condition in which the other parameters are at their center or zero value). a $\mathrm{CH}$ versus aMCI standard-baseline. b CH versus aMCI robust-baseline. c CH versus aMCI robust-multiwave.

group across all 4 factors. Figure $3 \mathrm{~b}$ depicts the nonsignificant aMCI $\times$ age interaction for Verbal Learning and Memory; the age-related slopes of the $\mathrm{CH}$ and $\mathrm{aMCI}$ robust groups were -0.014 and -0.002 , respectively. The interaction was removed and rerun to obtain the other parameter estimates shown in table 2.

For the aMCI robust-multiwave analyses, there were significant aMCI $\times$ age interactions for both memory factors. Follow-up analyses indicated that those in the aMCI robustmultiwave group were lower on average and declined significantly faster than their $\mathrm{CH}$ peers on Immediate Memory (simple age slopes of -0.044 SDs/year for the aMCI robust-multiwave group vs. -0.020 SDs/year for the CH group). Patterns were similar in the Verbal Learning and Memory factor, with age slopes of $-0.015 \mathrm{SDs} /$ year for the $\mathrm{CH}$ group and $-0.037 \mathrm{SDs} /$ year for the aMCI robust-multiwave group, as shown in figure 3c. The $95 \%$ CIs for the age-related changes per year in Verbal Learning and Memory z-scores were -0.003 to 0.058 for aMCI standard-baseline, -0.019 to -0.004 for aMCI robust-baseline, and -0.056 to -0.018 for aMCI robust-multiwave.

Secondary $\chi^{2}$ analyses identified no significant associations between family history and aMCI status or APOE and aMCI status across any of the $3 \mathrm{aMCI}$ methods. Longitudinal analyses did not reveal any significant family history or APOE main effects or interactions with age in any of the 4 cognitive factors among participants who had completed 3 waves of assessment. 
Koscik et al.: Emergence of Mild Cognitive Impairment in Late Middle-Aged Adults in the Wisconsin Registry for Alzheimer's Prevention

\section{Discussion}

One of the highest priorities in AD research is to find indicators of risk that are reliable and valid early in the lifespan when interventions to alter the disease course are likely to be most effective. Our study builds on previous work on MCI conducted primarily with older samples, extending categorization of mild cognitive deficit into a normally functioning middleaged group. We focused on cognitive outcomes, particularly in episodic memory, in the belief that subtle variations in performance and patterns of decline may prove significant and may contribute meaningfully to the search for preclinical markers of AD susceptibility.

The standard approach to MCI identification, in which baseline memory performance was compared to published age norms, identified $4.5 \%$ of WRAP participants as having aMCI. This is similar to MCI prevalence estimates in 2 prior studies with samples in their early $60 \mathrm{~s}$ $[15,43]$, both of which found a $4 \%$ rate of aMCI based on similar methods. However, in our study, only $50 \%$ of persons with aMCI at baseline relative to published norms met criteria for multiwave aMCI. More importantly, the simple age slopes showed that instead of declining over time on the crucial Verbal Learning and Memory factor, the aMCI group identified by standard criteria showed improvement in learning and memory performance over the 6-year follow-up period. Two prior studies with middle-aged samples have also shown relatively low rates of stability for standard MCI diagnoses over follow-ups of a few years [15, 43], whereas a third study, which combined middle-aged and elderly subjects in stability analyses, showed greater longitudinal consistency in MCI classification [17]. These findings for middleaged samples are similar to what has been observed in population-based (i.e. nonclinic) studies of elderly groups $[14,42,46]$. For older adults in the community diagnosed with MCI based on a single assessment, the most likely outcomes over a few years are either stable low performance or reversion to normal-range scores. Although only a relatively small percentage progress to $\mathrm{AD}$, the rate of progression is nonetheless greater than that for persons with normal cognitive performance at study onset [14].

Using robust norms to identify low baseline memory performance resulted in more than twice as many persons (13.7\% of the sample) being identified as having aMCI, and $60 \%$ of these individuals meeting aMCI robust criteria also met criteria for multiwave aMCI. However, for the aMCI robust-baseline group as a whole, there was no significant longitudinal change across the 6-year follow-up on any of the cognitive factors. While the use of robust norms can help to minimize oversight of significant cognitive deficit, which can be particularly likely in persons with high education or high ability [40,41], our data suggest that the application of robust norms at a single point in time could lead to identification of persons with mild memory and executive function difficulties that do not progress in a consistent manner in the midlife phase over the time span that subjects were followed here. Overall, it appears that neither of the approaches that used baseline data alone are optimal for identifying persons with subtle but reliable declines in episodic memory that may be the earliest cognitive indication of AD.

Using criteria that capitalized on longitudinal cognitive data, we found that nearly $12 \%$ of this late middle-aged sample have memory deficits consistent with aMCI. By requiring performance to be below expected levels on at least 2 of 3 testing occasions, the goal was to enhance the likelihood of identifying persons with relatively persistent memory problems who, despite normal activities of daily living function, may be at increased risk for cognitive decline and possible progression to $\mathrm{AD}$. The longitudinal factor score data suggest that may be the case. The multiwave aMCI group declined more over the 6 years of follow-up than the $\mathrm{CH}$ group on both factors related to episodic memory; the age-related decline in the aMCI multiwave group was also more than either of the other 2 aMCI groups, as shown by the largely nonoverlapping CIs for the aMCI simple slopes. Executive function, while lower overall 
compared to the $\mathrm{CH}$ group, did not significantly decline over time in the multiwave aMCI group.

The aMCI robust-multiwave group most closely approximates cognitive criteria for MCI due to $\mathrm{AD}$ as recommended by the National Institutes of Health (NIH) workgroup, and it improves the sensitivity of detection of subtle decline by its use of internal norms that adjust for the high literacy and education of the sample. This multiwave group exhibited relatively persistent episodic memory deficit for age and literacy level, and they showed an accelerated rate of episodic memory decline across a period of 6 years. Despite these memory difficulties, they were typically independent in everyday function based on reports of close informants, and global cognitive performance as measured by the MMSE remained intact. In contrast to the workgroup criteria for MCI due to AD, however, WRAP participants with multiwave aMCI were not specifically selected for concern about memory decline.

A somewhat similar approach to MCI classification was taken by Collie et al. [47], who required impairment on word list learning on 3 consecutive testings, at 6-month intervals, for MCI classification. Compared to matched controls with normal word list learning on all testings, those with stable MCI performed worse on additional memory tests administered after the third test wave. Compared to WRAP, the Collie et al. [47] study was based on a smaller sample, included a greater proportion of elderly participants, and used much shorter test-retest intervals. However, their conclusion was similar to ours, i.e. that serial assessments are crucial for differentiating individuals with $\mathrm{MCI}$ from $\mathrm{CH}$ persons and from those with transient cognitive impairments. An accurate classification of $\mathrm{MCI}$ is needed to identify those at risk for $\mathrm{AD}$ and is particularly important in studies of preclinical $\mathrm{AD}$, like WRAP, which are attempting to define the earliest neurobiology of $\mathrm{AD}$ in preparation for prevention clinical trials.

A concern for all methods of MCI identification is that criteria will select persons with characteristically weak memory performance unrelated to AD risk (so called 'accidental MCI') $[48,49]$. In our data, the likelihood that this may have occurred appears strongest for the aMCI standard group, where baseline performance was lower on all cognitive factors compared to the $\mathrm{CH}$ group; premorbid performance, estimated from demographics and reading scores, was also significantly lower in the aMCI standard group for all cognitive factors. By contrast, for the multiwave aMCI group, estimated premorbid ability on secondary memory factors was comparable to that of the $\mathrm{CH}$ group, and the accelerated rate of decline in memory performance after baseline suggests a progressively deteriorating memory pattern. Using quantitative estimation of premorbid memory ability as a tool for identifying aMCI has also been supported by other recent research [50].

Parental family history of AD and APOE genotype were not associated with aMCI classification in these analyses. Biomarker evidence for preclinical AD has been reported among asymptomatic offspring of persons with AD by several research groups (e.g. Donix et al. [51]; Xiong et al. [52]), including our own (e.g. Johnson et al. [53]; Okonkwo et al. [10]), and we have also found evidence for subtle differences in memory strategies for persons with and without a family history of AD $[8,9]$. The absence of a significant family history effect may be due in part to the relatively small number of subjects meeting aMCI criteria and due to the fact that relatively few family history participants had completed 3 waves of testing at the time these analyses were conducted. There was also no evidence for elevated rates of aMCI in persons with at least $1 \mathrm{APOE} \varepsilon 4$ allele. In addition to the small aMCI group limiting power to detect APOE effects, age and zygosity may have been factors in this negative finding. A recent metaanalysis of studies of APOE effects on cognition in nonclinical samples [54] concluded that having an $\varepsilon 4$ allele is associated with a modest negative influence on episodic memory, but that the effect size is smaller in younger samples and for $\varepsilon 4$ heterozygotes as opposed to homozygotes. The WRAP sample is relatively young and only $11.7 \%$ of our APOE-positive subgroup had $2 \varepsilon 4$ alleles. 
Study limitations include the following: although word list learning and recall tests are among the most commonly recommended measures for detecting memory loss related to $\mathrm{MCI}$ and $\mathrm{AD}, \mathrm{aMCI}$ categorization in our sample may have differed if longitudinal data from different memory tasks, or multiple memory measures, had been available. We were not able to verify the psychometric diagnoses with clinical exams since clinical diagnostic examinations were not a part of WRAP procedures in these early test waves. The relatively small number of subjects with aMCI limits statistical power to test associations with family history, APOE, or other predictors. Finally, although beyond the scope of this paper, it is important to recognize that we do not yet have biomarker evidence to indicate that aMCI is likely due to $\mathrm{AD}$, nor do we know as yet whether participants with aMCI will progress to clinical AD.

Important future steps for WRAP will be to repeat these analyses when a larger proportion of the baseline sample has completed 3 waves of assessment. Subsequent analyses will also compare the multiwave approach with other methods of evaluating cognitive change, such as a reliable change index approach (e.g. Stein et al. [55]), and analysis of whether practice effects in early waves predict subsequent aMCI status in ways similar to other studies (e.g. Duff et al. [56]). It will also be critical to compare biomarker profiles for the aMCI robustmultiwave versus $\mathrm{CH}$ groups and to continue longitudinal follow-up to further assess cognitive trajectories and to establish dementia endpoints. The validity of the current multiwave aMCI classification will be supported if the aMCI group shows neuroimaging and/or CSF profiles consistent with preclinical AD, and ultimately, higher rates of AD. Equally important will be the characterization of factors (genetic, familial, and health- and lifestyle-related) that are associated with the earliest signs of aMCI, or alternatively, with remaining dementia free.

\section{Acknowledgements}

The authors gratefully acknowledge the assistance of the WRAP scheduling, assessment, and project management staff. We especially thank the WRAP participants. This research was supported by NIA grant R01AG27161 (Wisconsin Registry for Alzheimer Prevention: Biomarkers of Preclinical AD), NIH grant M01RR03186 (University of Wisconsin Clinical and Translational Research Core), the Helen Bader Foundation, Northwestern Mutual Foundation, and Extendicare Foundation. The project described was also supported by the Clinical and Translational Science Award (CTSA) program, through the NIH National Center for Advancing Translational Sciences (NCATS), grant UL1TR000427. The content is solely the responsibility of the authors and does not necessarily represent the official views of the NIH.

\section{References}

- 1 Albert MS, Moss MB, Tanzii R, Jones K: Preclinical prediction of AD using neuropsychological tests. J Int Neuropsychol Soc 2001; 7:631-639.

2 Backman L, Jones S, Berger AK, Laukka EJ, Small BJ: Cognitive impairment in preclinical Alzheimer's disease: a meta-analysis. Neuropsychology 2005;19:520-531.

3 Petersen RC, Smith GE, Waring SC, Ivnik RJ, Tangalos EG, Kokmen E: Mild cognitive impairment: clinic characterization and outcome. Arch Neurol 1999;56:303-308.

- 4 Winblad B, Palmer K, Kivipelto M, Jelic V, Gratiglioni L, Wahlund LO, Nordberg A, Backman L, Albert M, Almkvist O, et al: Mild cognitive impairment: beyond controversies, towards a consensus-report of the International Working Group on Mild Cognitive Impairment. J Intern Med 2004;256:240-246.

- 5 Albert MS, DeKosky ST, Dickson D, Dubois B, Feldman HH, Fox NC, Gamst A, Holtzman DM, Jagust WJ, Peterson RC, et al: The diagnosis of mild cognitive impairment due to Alzheimer's disease: recommendations from the National Institute on Aging-Alzheimer's Association workgroups on diagnostic guidelines for Alzheimer's disease. Alzheimers Dement 2011;7:270-279.

6 Jack CR Jr, Vemuri P, Wiste HJ, Weigand SD, Aisen PS, Trojanowski JQ, Shaw LM, Bernstein MA, Peterson RC, Weiner MW, et al: Evidence for ordering of Alzheimer disease biomarkers. Arch Neurol 2011;68:1526-1535.

7 Debette S, Wolf PA, Beiser A, Au R, Himali JJ, Pikula A, Auerbach S, Decarli C, Seshadri S: Association of parental dementia with cognitive and brain MRI measures in middle-aged adults. Neurology 2009;73:2071-2078. 
Koscik et al.: Emergence of Mild Cognitive Impairment in Late Middle-Aged Adults in the Wisconsin Registry for Alzheimer's Prevention

- 8 La Rue A, Hermann BP, Jones JE, Johnson S, Asthana S, Sager MA: Effect of parental family history of Alzheimer's disease on serial position profiles. Alzheimers Dement 2008;4:285-290.

9 Chang TS, Coen MH, La Rue A, Jonaitis E, Koscik RL, Hermann B, Sager MA: Machine learning amplifies the effect of parental family history of Alzheimer's disease on list learning strategy. J Int Neuropsychol Soc 2012; 18:428-439.

-10 Okonkwo O, Xu G, Dowling NM, Bendlin B, La Rue A, Hermann BP, Koscik R, Jonaitis E, Rowley H, Carlsson CM, et al: Family history of Alzheimer disease predicts hippocampal atrophy in healthy middle-aged adults. Neurology 2012;78:1769-1776.

11 Elias MF, Beiser A, Wolf PA, Au R, White RF, D'Agostino RB: The preclinical phase of Alzheimer's disease: a 22-year prospective study of the Framingham Cohort. Arch Neurol 2000;57:808-813.

12 Kawas CH, Corrada MM, Brookmeyer R, Morrison A, Resnick SM, Zonderman AB: Visual memory predicts Alzheimer's disease more than a decade before diagnosis. Neurology 2003;60:1089-1093.

13 Jak AJ, Bondi MW, Delano-Wood L, Wierenga C, Corey Bloom J, Salmon DP, Delis DC: Quantification of five neuropsychological approaches to defining mild cognitive impairment. Am J Psychiatry 2009;17:368-375.

14 Ganguli M, Snitz BE, Saxton JA, Chang CC, Lee CW, Vander Bilt J, Hughes TF, Loewenstein DA, Unverzagt FW, Peterson RC: Outcomes of mild cognitive impairment by definition. Arch Neurol 2011;68:761-767.

15 Anstey KJ, Cherbuin N, Christensen H, Burns R, Reglade-Meslin C, Salim A, Kumar R, Jorm AF, Sachdev P: Follow-up of mild cognitive impairment and related disorders over four years in adults in their sixties: the Path Through Life Study. Dement Geriatr Cogn Disord 2008;26:226-233.

16 Schonknecht P, Pantel J, Kruse A, Schroder J: Prevalence and natural course of aging-associated cognitive decline in a population-based sample of young-old subjects. Am J Psychiatry 2005;162:2071-2077.

17 Dolcos S, MacDonald SW, Braslavsky A, Camicioli R, Dixon RA: Mild cognitive impairment is associated with selected functional markers: integrating concurrent, longitudinal, and stability effects. Neuropsychology 2012;26:209-223.

18 Anstey KJ, Cherbuin N, Eramudugolla R, Sargent-Cox K, Easteal S, Kumar R, Sachdev P: Characterizing mild cognitive disorders in the young-old over 8 years: prevalence, estimated incidence, stability of diagnosis, and impact on IADLs. Alzheimers Dement 2013;9:640-648.

-19 Sager MA, Hermann BP, La Rue A: Middle-aged children of persons with Alzheimer's disease: APOE genotypes and cognitive function in the Wisconsin Registry for Alzheimer's Prevention. J Geriatr Psychiatry Neurol 2005; 18:245-249.

20 Schmidt M: Rey Auditory Verbal Learning Test: A Handbook. Los Angeles, Western Psychological Services, 1996.

-21 DeSanti S, Pirraglia E, Barr W, Babb J, Williams S, Rogers K, Glodzik L, Brys M, Mosconi L, Reisberg B, et al: Robust and conventional neuropsychological norms: diagnosis and prediction of age-related cognitive decline. Neuropsychology 2008;22:469-484.

-22 McKhann G, Drachman D, Folstein M, Katzman R, Price D, Stadlan EM: Clinical diagnosis of Alzheimer's disease: report of the NINCDS-ADRDA Work Group under the auspices of Department of Health and Human Services Task Force on Alzheimer's Disease. Neurology 1984;34:939-944.

23 Folstein MF, Folstein SE, McHugh PR: 'Mini-mental state'. A practical method for grading the cognitive state of patients for the clinician. J Psychiatr Res 1975;12:189-198.

24 Wechsler D: Wechsler Abbreviated Scale of Intelligence. San Antonio, The Psychological Corporation, 1999.

25 Lawton MP, Brody EM: Assessment of older people: self-maintaining and instrumental activities of daily living. Gerontologist 1969;9:179-186.

26 Radloff L: The CES-D scale: a self-report depression scale for research in the general population. Appl Psychol Meas 1977;1:385-401.

27 Grice JW: Computing and evaluating factor scores. Psychol Methods 2001;6:430-450.

28 Lezak M, Howieson D, Loring D: Neuropsychological Assessment, ed 4. New York, Oxford University Press, 2004.

29 Wechsler D: Wechsler Adult Intelligence Scale. San Antonio, Psychological Corporation, 1997.

-30 Dowling NM, Hermann B, La Rue A, Sager MA: Latent structure and factorial invariance of a neuropsychological test battery for the study of preclinical Alzheimer's disease. Neuropsychology 2010;24:742-756.

31 Jonaitis E, La Rue A, Mueller K, Koscik R, Hermann B, Sager MA: Cognitive activities and cognitive performance in middle-aged adults at risk for Alzheimer's disease. Psychol Aging, in press.

-32 Barona A, Reynolds CR, Chastain R: A demographically based index of premorbid intelligence for the WAIS-R. J Consult Clin Psychol 1984;52:885-887.

33 Crawford JR, Stewart LE, Parker DM, Besson JAO, Cochrane RHB: Estimation of premorbid intelligence: combining psychometric and demographic approaches improves predictive accuracy. Pers Individ Diff 1989; 10:793-796.

34 Griffin SL, Mindt MR, Rankin EJ, Ritchie AJ, Scott JG: Estimating premorbid intelligence: comparison of traditional and contemporary methods across the intelligence continuum. Arch Clin Neuropsychol 2011;17:497507.

35 Duff K: Predicting premorbid memory functioning in older adults. Appl Neuropsychol 2010;17:278-282.

36 Duff K, Chelune GJ, Dennett K: Predicting estimates of premorbid memory functioning: validation in a dementia sample. Arch Clin Neuropsychol 2011;26:701-705. 
-37 Manly JJ, Jacobs DM, Touradji P, Small SA, Stern Y: Reading level attenuates differences in neuropsychological test performance between African American and White elders. J Int Neuropsychol Soc 2002;8:341-348.

-38 Carlozzi NE, Stout JC, Mills JA, Duff K, Beglinger LJ, Aylward EH, Whitlock KB, Solomon AC, Queller S, Langbehn DR, et al: Estimating premorbid IQ in the prodromal phase of a neurodegenerative disease. Clin Neuropsychol 2011;25:757-777.

39 Wilkinson G: Wide Range Achievement Test - Revision 3. Wilmington, Jastak Association, 1993.

40 Sliwinski MJ, Hofer SM, Hall C, Buschke H, Lipton RB: Modeling memory decline in older adults: the importance of preclinical dementia, attrition, and chronological age. Psychol Aging 2003;18:658-671.

-41 Tuokko H, Garrett DD, McDowell I, Silverberg N, Kristiansson B: Cognitive decline in high-functioning older adults: reserve or ascertainment bias? Aging Ment Health 2003;7:259-270.

$>42$ Bruscoli M, Lovestone S: Is MCI really just early dementia? A systematic review of conversion studies. Int Psychogeriatr 2004;16:129-140.

43 Cherbuin N, Reglade-Meslin C, Kumar R, Jacomb P, Easteal S, Christensen H, Sachdev P, Anstey KJ: Risk factors of transition from normal cognition to mild cognitive disorder: the Path Through Life Study. Dement Geriatr Cogn Disord 2009;28:47-55.

-44 Willett J, Singer J, Martin N: The design and analysis of longitudinal studies of development and psychopathology in context: statistical models and methodological recommendations. Dev Psychopathol 1998;10:395426.

45 Aiken LS, West SG: Multiple Regression: Testing and Interpreting Interactions. London, Sage Publications, 1991.

46 Palmer K, Backman L, Winblad B, Fratiglioni L: Mild cognitive impairment in the general population: occurrence and progression to Alzheimer disease. Am J Geriatr Psychiatry 2008;16:603-611.

47 Collie A, Maruff P, Currie J: Behavioral characterization of mild cognitive impairment. J Clin Exp Neuropsychol 2002;24:720-733.

48 Brooks BL, Iverson GL, White T: Substantial risk of 'accidental MCI' in healthy older adults: base rates of low memory scores in neuropsychological assessment. J Int Neuropsychol Soc 2007;13:490-500.

-49 Palmer BW, Boone KB, Lesser IM, Wohl MA: Base rates of 'impaired' neuropsychological test performance among healthy older adults. Arch Clin Neuropsychol 1998;13:503-511.

50 Murayama N, Tagaya H, Ota K, Fujishiro H, Manabe Y, Sato K, Isek E: Neuropsychological detection of the early stage of amnestic mild cognitive impairment without objective memory impairment. Dement Geriatr Cogn Disord 2013;35:98-105.

-51 Donix M, Burggren AC, Suthana NA, Siddarth P, Ekstrom AD, Krupa AK, Jones M, Martin-Harris L, Ercoli LM, Miller KJ, et al: Family history of Alzheimer's disease and hippocampal structure in healthy people. Am J Psychiatry 2010;167:1399-1406.

52 Xiong C, Roe CM, Buckles V, Fagan A, Holtzman DM, Balota D, Duchek J, Storandt M, Mintun M, Grant E, et al: Role of family history for Alzheimer biomarker abnormalities in the adult children study. Arch Neurol 2011; 68:1311-1317.

-53 Johnson SC, Schmitz TW, Trivedi MA, Ries ML, Torgerson BM, Carlsson CM, Asthana S, Hermann BP, Sager MA: The influence of Alzheimer disease family history and apolipoprotein E epsilon 4 on mesial temporal lobe activation. J Neurosci 2006;26:6069-6076.

54 Wisdom NM, Callahan JL, Hawkins KA: The effects of apolipoprotein E on non-impaired cognitive functioning: a meta-analysis. Neurobiol Aging 2011;32:63-74.

55 Stein J, Luppa M, Maier W, Tebarth F, Heser K, Scherer M, Zimmermann T, Eisele M, Bickel H, Mosch E, et al: The assessment of changes in cognitive functioning in the elderly: age- and education-specific reliable change indices for the SIDAM. Dement Geriatr Cogn Disord 2012;33:73-83.

-56 Duff K, Beglinger LJ, Van Der Heiden S, Moser DJ, Arndt S, Schultz SK, Paulsen JS: Short-term practice effects in amnestic mild cognitive impairment: implications for diagnosis and treatment. Int Psychogeriatr 2008;20: 986-999. 